\title{
Charged Dark Matters and Extended Standard Model
}

\author{
Jae-Kwang Hwang \\ JJJ Physics Laboratory, Brentwood, TN 37027, USA \\ E-mail: jkhwang.koh@gmail.com
}

\begin{abstract}
The properties of the charged dark matters are discussed in terms of the new three-dimensional quantized space model. Because of the graviton evaporations, the very small Coulomb's constant $(\mathrm{k}(\mathrm{dd}))$ of $10^{-48} \mathrm{k}$ and large gravitation constant $\left(\mathrm{G}_{\mathrm{N}}(\mathrm{dd})\right)$ of $10^{6} \mathrm{G}_{\mathrm{N}}$ for the charged dark matters at the present time are expected. The tentative values of $\mathrm{G}$ and $\mathrm{k}$ are used for the explanation purpose. Therefore, $\mathrm{F}_{\mathrm{c}}(\mathrm{mm})>\mathrm{F}_{\mathrm{g}}(\mathrm{dd})>\mathrm{F}_{\mathrm{g}}(\mathrm{mm})>\mathrm{F}_{\mathrm{g}}(\mathrm{dm})$ $>\mathrm{F}_{\mathrm{c}}(\mathrm{dd})>\mathrm{F}_{\mathrm{c}}(\mathrm{dm})=0$ for the proton-like particle. Also, the gravitation constant has been changed with increasing of the time because of the graviton evaporation. In the present work, the B1, B2 and B3 bastons with the condition of $\mathrm{k}(\mathrm{mm})=\mathrm{k}>\mathrm{k}(\mathrm{dd})>\mathrm{k}(\mathrm{dm})=0$ are explained as the good candidates of the dark matters. Also, the particle creation, dark matters and dark energy could be deeply associated with the changing gravitation constants $(\mathrm{G})$. It is expected that the changing process of the gravitation constant between the matters from $\mathrm{G}_{\mathrm{N}}(\mathrm{mm}) \approx 10^{36} \mathrm{G}_{\mathrm{N}}$ to $\mathrm{G}_{\mathrm{N}}(\mathrm{mm})=\mathrm{G}_{\mathrm{N}}$ happened mostly near the inflation period. Therefore, during most of the universe evolution the gravitation constant could be taken as $\mathrm{G}_{\mathrm{N}}\left(\mathrm{mm}_{\mathrm{m}}\right)=$ $\mathrm{G}_{\mathrm{N}}$. And the effective charges and effective rest masses of the particles are defined in terms of the fixed Coulomb's constant (k) and fixed gravitation constant $\left(\mathrm{G}_{\mathrm{N}}\right)$. Then, the effective charge of the B1 dark matter with $\mathrm{EC}=-\frac{2}{3} \mathrm{e}$ is $(\mathrm{EC})_{\mathrm{eff}}=-\frac{2}{3} 10^{-24} \mathrm{e}$.
\end{abstract}

Key words: charged dark matters, gravitation constant, Coulomb's constant, dark energy density, particle creation, extended standard model

\section{Introduction}

The dark matters have been known to have two properties. First the electromagnetic interactions between the dark matters (d) and normal matters (m) are zero. Secondly, the electromagnetic interactions between the dark matters are zero. Therefore, the zero Coulomb's forces of $F_{c}(d m)=0$ and $F_{c}(d d)=0$ have been proposed. Here, $d$ and $m$ represent the dark matter and normal matter, respectively. Because of the zero Coulomb's force, the electrically neutral particles have been proposed as the most possible candidates of the dark matters. In other words, the electric charges (EC) of these dark matters are zero in $\mathrm{F}_{\mathrm{c}}(\mathrm{EC})=\mathrm{k} \frac{E C 1 E C 2}{r^{2}}$. Also, the mini-charged particles (or milli-charged particles) with the near-zero EC charge [1] have been proposed as other possible candidates of the dark matters which give the very small Coulomb's forces between the dark matters and normal matters and between the dark matters. In this case, the same Coulomb's constant of $\mathrm{k}$ is applied for both of dark matters and normal matters.

In the present work, the zero Coulomb's constant of $\mathrm{k}(\mathrm{dm})=0$ is applied between the dark matters and normal matters in order to meet the first condition. In order to meet the second condition, the very small $\mathrm{k}(\mathrm{dd})$ values for the dark matters and the $\mathrm{k}(\mathrm{mm})=\mathrm{k}$ values for normal matters are proposed. Therefore, the relation of $\mathrm{k}(\mathrm{mm})=\mathrm{k}>>\mathrm{k}(\mathrm{dd})>$ $\mathrm{k}(\mathrm{dm})=0$ is shown in Figs. 1 and 2. In this case, the dark matters can have the EC charges close to the EC charge of the electron. The B1, B2 and B3 bastons with the tentative electric charges of $-2 / 3 e,-5 / 3 e$ and $-8 / 3 e$, respectively, were, for the first time, reported as the possible candidates of the dark matters in Ref. [2]. Therefore, in the present work, the $\mathrm{B} 1, \mathrm{~B} 2$ and B3 bastons with the condition of $\mathrm{k}(\mathrm{mm})=\mathrm{k}>\mathrm{k}(\mathrm{dd})>\mathrm{k}(\mathrm{dm})=0$ are explained as the good candidates of the dark matters. The relations of $\mathrm{G}_{\mathrm{N}}(1 \mathrm{l})=\mathrm{G}_{\mathrm{N}}(\mathrm{qq})=\mathrm{G}_{\mathrm{N}}(\mathrm{mm})$ and $\mathrm{k}(1 \mathrm{l})=\mathrm{k}(\mathrm{qq})=\mathrm{k}(\mathrm{mm})=\mathrm{k}$ are assumed in Fig. 1. Here, 1 and q represent the leptons and quarks, respectively. Then the normal matters consist of leptons, quarks and hadrons and the dark matters are the B1, B2 and B3 bastons [2]. In Figs. 1 and 2, the Coulomb's constant (k) and gravitation constant $(\mathrm{G})$ have been changed in terms of the conserved charges and conserved rest masses of the particles. And the effective charges and effective rest masses of the particles are defined in terms of the fixed Coulomb's constant $(\mathrm{k})$ and fixed gravitation constant $\left(\mathrm{G}_{\mathrm{N}}\right)$. Then, the effective charge of the $\mathrm{B} 1$ dark matter with $\mathrm{EC}=-\frac{2}{3}$ e is $(\mathrm{EC})_{\mathrm{eff}}=-\frac{2}{3} 10^{-24} \mathrm{e}$. And it is concluded that, at 2-3 $10^{8}$ years after big bang, the large effective rest masses of the baryons could explain the cooler gas temperature measured by the EDGE experiments.

In Fig. 2, the new concepts of the photon confinement and graviton evaporation are introduced. The Coulomb's constant should be constant with increasing of the time because of the photon confinement. The gravitation constant has been changed since the big bang because of the graviton evaporation. In the present work, the particle creation, dark matters and dark energy could be deeply associated with the changing gravitation constants $(\mathrm{G})$. It is shown that the relation of, at the present time, $F_{c}(\mathrm{~mm})>F_{g}(d d)>F_{g}(m m)>F_{g}(d m)>F_{c}(d d)>F_{c}(d m)=0$ for the proton-like particle could explain the universe evolution including the B1, B2 and B3 dark matters by giving the tentative values of the Coulomb's constants $(\mathrm{k})$ and gravitation constants $(\mathrm{G})$ for the explanation purpose in Fig. 1. It is discussed that the gravitational constant $\left(\mathrm{G}_{\mathrm{N}}(\mathrm{mm})\right)$ could be decreased from the very large value like $10^{116} \mathrm{G}_{\mathrm{N}}$ down to the very small value like $\mathrm{G}_{\mathrm{N}}$ 
near the inflation period in Fig. 1 and Table 1. Therefore, during most of the universe evolution the gravitation constant could be taken as $\mathrm{G}_{\mathrm{N}}(\mathrm{mm})=\mathrm{G}_{\mathrm{N}}$. The relations between particles, Planck energies and gravitation constants are

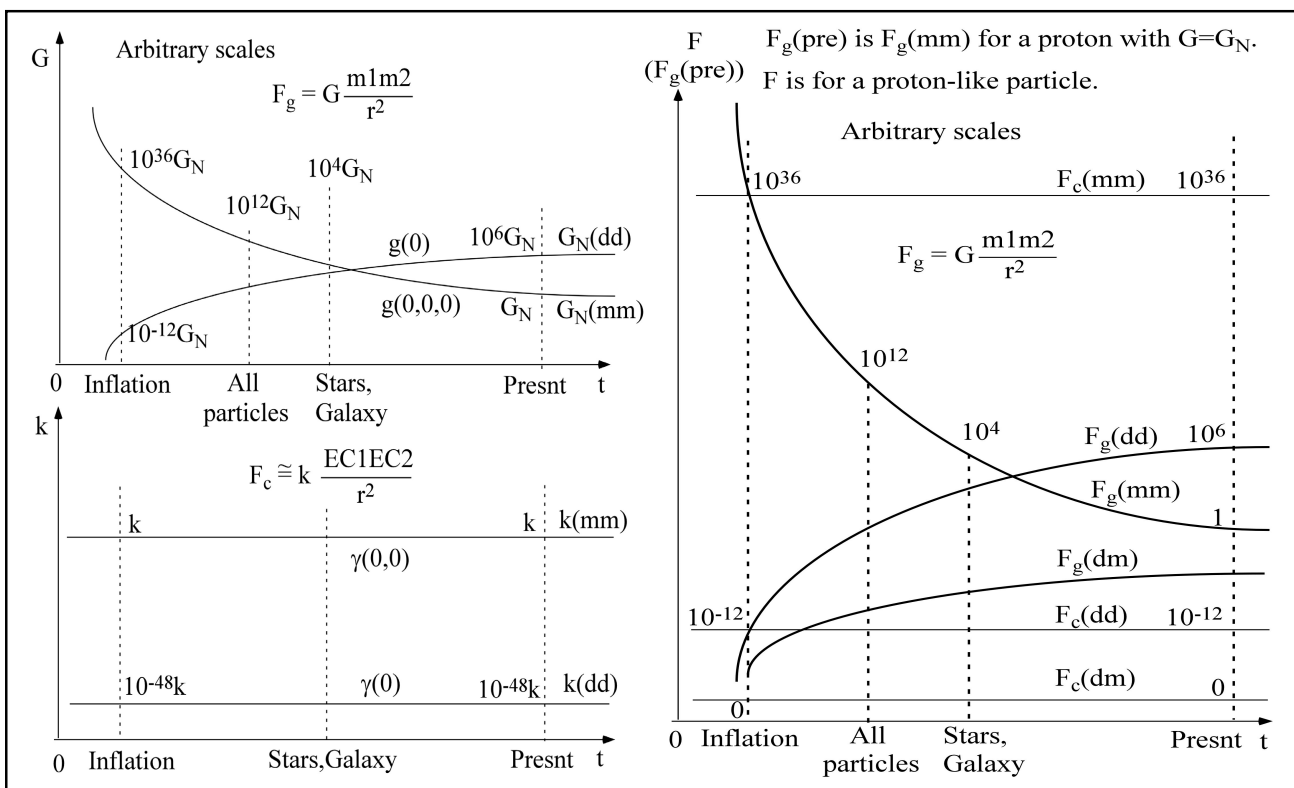

Fig. 1. The tentative values of $\mathrm{G}$ and $\mathrm{k}$ are used for the explanation purpose (see Fig. 2 and

Table 1$). \mathrm{F}_{\mathrm{c}}(\mathrm{mm}) \approx 10^{36} \mathrm{~F}_{\mathrm{g}}(\mathrm{mm})$ for the proton at the present time.

tentatively shown. It is concluded that the photons and gravitons are the first two particles created since the big bang. The particles can be created from the decay of the matter universe and the pair production of the particle and antiparticle with decreasing of the gravitation constant $\left(\mathrm{G}_{\mathrm{N}}(\mathrm{mm})\right)$ in Table 1. And, the galaxy particles created through the decaying process of the matter universe should have the large black holes at the center and the particles at the outside like the Milky Way galaxy [9]. This is the reason why each galaxy has the large black hole at its center.

Table 1. The relations between particles, Planck energies and gravitation constants are shown. From the relation of $\mathrm{E}_{\mathrm{p}}$ $\geqq \mathrm{E}($ particle $)=10^{\mathrm{A}} \mathrm{eV}, \mathrm{A} \leq 28-\mathrm{x} / 2$. If $\mathrm{G}=10^{\mathrm{x}} \mathrm{G}_{\mathrm{N}}, \mathrm{E}_{\mathrm{p}}=(\hbar c / G)^{0.5}=10^{-\mathrm{x} / 2} 10^{28} \mathrm{eV}$. See Fig. 1 .

\begin{tabular}{ccc}
\hline \hline Particles [1] & $\mathrm{E}_{\mathrm{p}}(\mathrm{mm}),(\mathrm{eV})$ & $\mathrm{G}=\mathrm{G}_{\mathrm{N}}(\mathrm{mm})=10^{\times} \mathrm{G}_{\mathrm{N}}$ \\
\hline$\gamma$ & $\approx 0$ & $\approx \infty$, Big bang \\
Graviton & $10^{-30}$ & $10^{116} \mathrm{G}_{\mathrm{N}}$ \\
$v_{\mathrm{e}}$ & $10^{-2}$ & $10^{60} \mathrm{G}_{\mathrm{N}}$ \\
$\mathrm{B} 1$ & $10^{2}$ & $10^{52} \mathrm{G}_{\mathrm{N}}$ \\
e, u, d & $10^{6}$ & $10^{44} \mathrm{G}_{\mathrm{N}}$ \\
p, n & $10^{10}$ & $10^{36} \mathrm{G}_{\mathrm{N}} \approx$ Inflation \\
W(-1,0), Z(0,0), Baryons, B2 & $10^{11}$ & $10^{34} \mathrm{G}_{\mathrm{N}}$ \\
Le, Nuclei, Atoms, Gas & $10^{13}$ & $10^{30} \mathrm{G}_{\mathrm{N}}$ \\
All elementary particles & $10^{22}$ & $10^{12} \mathrm{G}_{\mathrm{N}}, \approx 10^{5}$ years \\
Stars, Galaxies & $10^{26}$ & $10^{4} \mathrm{G}_{\mathrm{N},} \approx 10^{8}$ years \\
Present Planck mass & $10^{28}$ & $\mathrm{G}_{\mathrm{N}}, \approx 10^{9}$ years \\
\hline \hline
\end{tabular}




\section{Charged dark matters and gravitation constant}

The baston dark matters have only the electric charges (EC) [2]. Then, the bastons can be described as (EC). For example, the B1 dark matter with the electric charge of $-2 \mathrm{e} / 3$ is defined as B1(-2/3). The dark photon of $\gamma(0)$ and the dark graviton of $\mathrm{g}(0)$ are associated with the bastons in Fig. 2. The leptons have the electric charges (EC) and lepton charges (LC). Then, the leptons can be described as (EC,LC) [2]. For example, the electron neutrino and electron are

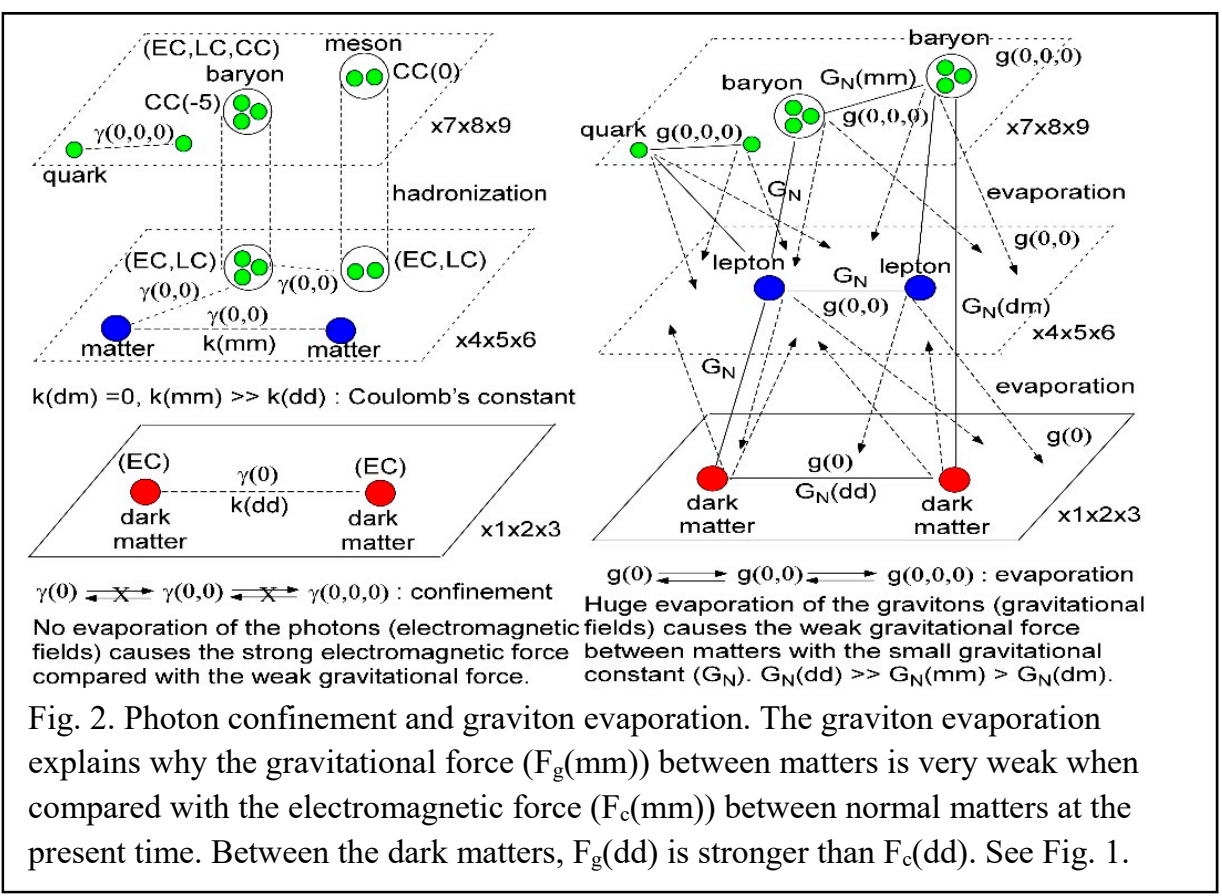

defined as $v_{\mathrm{e}}(0,-2 / 3)$ and $\mathrm{e}(-1,-2 / 3)$. The normal photon of $\gamma(0,0)$ and the graviton of $\mathrm{g}(0,0)$ are associated with the leptons in Fig. 2. The quarks have the electric charges (EC), lepton charges (LC) and color charges (CC). And the quarks can be described as (EC,LC,CC) [2]. For example, the $u$ and $d$ quarks are defined as $u(2 / 3,0, C C)$ and $d(-$ $1 / 3,0, \mathrm{CC})$. The photon of $\gamma(0,0,0)$ and the graviton of $\mathrm{g}(0,0,0)$ are associated with the quarks in Fig. 2 . Also, note that three charges of EC, LC and CC are tentatively quantized based on the systematics [2]. And, the baryons can be described as (EC,LC,-5) or (EC.LC) in Fig. 2 [2]. For example, the proton is defined as $(1,0,-5)$ or $(1,0)$. The fact that the baryons and mesons can be defined as $(\mathrm{EC}, \mathrm{LC})$ in the electromagnetic interactions is called as the hadronization in Fig. 2. Then, the hadrons can emit and absorb both photons of $\gamma(0,0)$ and $\gamma(0,0,0)$. This is why the charged baryons and charged mesons are interacting with the leptons like the electrons through the normal photons of $\gamma(0,0)$.

Three things have been observed for the electromagnetic interactions. First the electromagnetic interactions between the dark matters (d) and normal matters (m) are zero. Secondly, the electromagnetic interactions between the dark matters are zero. Thirdly, the independent quarks have never been observed electromagnetically through the normal photons of $\gamma(0,0)$. The third condition indicates that the electromagnetic interactions between the leptons and quarks are zero. And the first condition indicates that the electromagnetic interactions between the dark matters and normal matters are zero. This indicates that three photons of $\gamma(0), \gamma(0,0)$ and $\gamma(0,0,0)$ are not changed to each other. This is called as the photon confinement in the present work. This means that the different Coulomb force should be defined to the bastons, leptons and quarks in Fig. 2. Second condition can indicate that the $F_{c}(d d)$ value for the charged dark matters is nearly zero. So, the very small $\mathrm{k}(\mathrm{dd})$ value like $\mathrm{k}(\mathrm{dd})=10^{-48} \mathrm{k}$ for the charged B1, B2 and B3 dark matters can meet the second condition in Figs. 1 and 2.

Three things have been observed for the gravitational force at the present time. First, the gravitational force $\left(\mathrm{F}_{\mathrm{g}}(\mathrm{dm})\right)$ between dark matters and normal matters is not zero as seen in the galaxy structures. Secondly, the gravitational force $\left(F_{g}(\mathrm{~mm})\right)$ between the normal matters is very weak compared with the electromagnetic force $\left(\mathrm{F}_{\mathrm{c}}(\mathrm{mm})\right)$ between the 
normal matters. For example, for the proton $\mathrm{F}_{\mathrm{g}}(\mathrm{mm})=0.810^{-36} \mathrm{~F}_{\mathrm{c}}(\mathrm{mm})$. Thirdly, the gravitational force is dominating over the electromagnetic force for the dark matters. Because $F_{g}(\mathrm{~mm})$ is so small in the second condition, $F_{g}(d d)$ could be larger than $F_{g}(\mathrm{~mm})$ for the proton-like particle. And the gravitational force of $F_{g}(\mathrm{dm})$ could be smaller than the gravitational forces of $F_{g}(\mathrm{~mm})$ and $F_{g}(d d)$ because $F_{c}(d m)=0$ is smaller than the Coulomb's forces of $F_{c}(m m)$ and $F_{c}(d d)$. This gives the relations of $F_{g}(d d)>F_{g}(m m)>F_{g}(d m)>0$ and $G_{N}(d d)>G_{N}(m m)>G_{N}(d m)>0$ because the gravitational force formula is $\mathrm{F}_{\mathrm{g}}(\mathrm{dd})=\mathrm{G}_{\mathrm{N}}(\mathrm{dd}) \frac{m 1 m 2}{r^{2}}$. The non-zero gravitational force of $\mathrm{F}_{\mathrm{g}}(\mathrm{dm})$ indicates that three gravitons of $g(0), g(0,0)$ and $g(0,0,0)$ are changed to each other. This is called as the graviton evaporation in the present work in Fig. 1. These relations between the gravitational forces can be compared with the relations of $F_{c}(m m)>F_{c}(d d)$ $>\mathrm{F}_{\mathrm{c}}(\mathrm{dm})=0$ and $\mathrm{k}(\mathrm{mm})=\mathrm{k}>>\mathrm{k}(\mathrm{dd})>\mathrm{k}(\mathrm{dm})=0$. In order to make dark matters to be controlled by the attractive gravitational force in the third condition, the attractive gravitational force $\left(\mathrm{F}_{\mathrm{g}}(\mathrm{dd})\right)$ between the charged dark matters should be greater than the repulsive Coulomb's force $\left(\mathrm{F}_{\mathrm{c}}(\mathrm{dd})\right)$ between the charged dark matters. Therefore, $F_{g}(\mathrm{dd})>$ $F_{c}(d d)$. Therefore, the relation of $F_{c}(m m)>F_{g}(d d)>F_{g}(m m)>F_{g}(d m)>F_{c}(d d)>F_{c}(d m)=0$ for the proton-like particle is obtained as shown in Fig. 1.

But still why the gravitation force of $F_{g}(\mathrm{~mm})$ is so smaller than the electromagnetic force of $F_{c}(\mathrm{~mm})$ for the proton at the present time needs to be explained. In order to explain this question, we need to consider those forces near the inflation in Fig. 1. Because three photons of $\gamma(0), \gamma(0,0)$ and $\gamma(0,0,0)$ are not changed to each other, the Coulomb's constant of $\mathrm{k}(\mathrm{mm})_{\text {inf }}$ near the inflation should be equal to the Coulomb's constant of $\mathrm{k}(\mathrm{mm})_{\text {pre }}=\mathrm{k}$ at the present time. This means that $F_{c}(\mathrm{~mm})_{\text {inf }}$ is equal to $F_{c}(\mathrm{~mm})_{\text {pre }}$ for the proton. Because three gravitons of $g(0), g(0,0)$ and $g(0,0,0)$ are changed to each other, the gravitation constant of $\mathrm{G}_{\mathrm{N}}(\mathrm{mm})$ inf near the inflation can be greater than the gravitational constant of $\mathrm{G}_{\mathrm{N}}(\mathrm{mm})_{\text {pre }}=\mathrm{G}_{\mathrm{N}}$ at the present time because of the graviton evaporations. Near the inflation, $\mathrm{F}_{\mathrm{c}}(\mathrm{mm})_{\mathrm{inf}}$ could be equal or similar to $F_{g}(\mathrm{~mm})_{\text {inf }}$ for the proton. Then the $F_{c}(\mathrm{~mm})$ remains constant with increasing of the time since the inflation. However, $F_{g}(\mathrm{~mm})_{\text {inf }}$ near the inflation has been decreased to the present value of $F_{g}(\mathrm{~mm})_{\text {pre }}=F_{g}(\mathrm{~mm})$ for the proton with increasing of the time since the inflation in Fig. 1.

Because three photons of $\gamma(0), \gamma(0,0)$ and $\gamma(0,0,0)$ are not changed to each other, the Coulomb's constant of $k(d d)_{\text {inf }}$ near the inflation should be equal to the Coulomb's constant of $k(d d)_{\text {pre }}=k$ at the present time. This means that $F_{c}(d d)_{\text {inf }}$ is equal to $F_{c}(d d)$ pre for the proton-like particle. Because three gravitons of $g(0), g(0,0)$ and $g(0,0,0)$ are changed to each other, the gravitation constant of $\mathrm{G}_{\mathrm{N}}(\mathrm{dd})_{\text {inf }}$ near the inflation can be smaller than the gravitation constant of $\mathrm{G}_{\mathrm{N}}(\mathrm{dd})_{\text {pre }}$ at the present time because of the graviton evaporations. Near the inflation, $F_{c}(d d)_{\text {inf }}$ could be equal or similar to $F_{g}(d d)_{\text {inf }}$ for the proton-like particle. Then the $\mathrm{F}_{\mathrm{c}}(\mathrm{dd})$ remains constant with increasing of the time since the inflation. However, $\mathrm{F}_{\mathrm{g}}(\mathrm{dd})_{\text {inf }}$ near the inflation has been increased to the present value of $\mathrm{F}_{\mathrm{g}}(\mathrm{dd})_{\text {pre }}$ for the proton-like particle with increasing of the time since the inflation in Fig. 1.

Also, in Fig 2, the photons are confined within the corresponding space. This indicates that the Coulomb's constant (k) does not change since the inflation. Therefore, always $\mathrm{k}(\mathrm{mm})>>\mathrm{k}(\mathrm{dd})$ and $\mathrm{k}(\mathrm{mm})=\mathrm{k}$. However, the gravitation constant of $\mathrm{G}_{\mathrm{N}}$ is different because the gravitons can evaporate into other spaces in Fig. 2 . This indicates that the gravitation constant of $\mathrm{G}$ has been changing since the inflation. In other words, near the inflation period, $\mathrm{F}_{\mathrm{g}}(\mathrm{mm})>>$ $F_{g}(d d)$ and $F_{c}(m m) \gg F_{c}(d d)$ with the condition of $G_{N}(m m) \gg G_{N}(d d)$ and $k(m m)>>k(d d)$ in Figs. 1 and 2 . Then, because of the graviton evaporation, $\mathrm{G}_{\mathrm{N}}(\mathrm{mm})$ has been decreased and $\mathrm{G}_{\mathrm{N}}(\mathrm{dd})$ has been increased since the inflation. At the present time, $F_{g}(d d)>F_{g}(m m), F_{g}(d d)>F_{c}(d d)$ and $F_{g}(m m) \ll F_{c}(m m)$ with the condition of $G_{N}(m m)<G_{N}(d d)$ and $k(\mathrm{~mm})>>k(d d)$ in Fig. 1. At the present time, $F_{c}(m m)>F_{g}(d d)>F_{c}(d d)$ in the force strength and $F_{g}(d d)>$ $\mathrm{F}_{\mathrm{g}}(\mathrm{mm})$. In other words, it is assumed that $\mathrm{G}_{\mathrm{N}}(\mathrm{dd})>\mathrm{G}_{\mathrm{N}}(\mathrm{mm})=\mathrm{G}_{\mathrm{N}}=\mathrm{G}_{\mathrm{N}}(\mathrm{qq}) \approx \mathrm{G}_{\mathrm{N}}(1 \mathrm{l})$.

The tentative numerical values of $\mathrm{k}$ and $\mathrm{G}$ in Fig. 1 are added just in order to show that the graviton evaporation and photon confinement can explain the relative force strengths of the electromagnetic interactions and gravitational interactions well. For example, it can be assumed that near the inflation $\mathrm{G}_{\mathrm{N}}(\mathrm{mm}) \approx 10^{36} \mathrm{G}_{\mathrm{N}}$, and $\mathrm{G}_{\mathrm{N}}(\mathrm{dd})=10^{-12} \mathrm{G}_{\mathrm{N}}$ in Fig. 1. This condition indicates that the matters forms the black holes with the very large Schwarzschild radius because of the large gravitation constant of $\mathrm{G}_{\mathrm{N}}(\mathrm{mm}) \approx 10^{36} \mathrm{G}_{\mathrm{N}}$. Here Schwarzschild radius $\left(\mathrm{R}_{\mathrm{s}}\right)$ is $\mathrm{R}_{\mathrm{s}}=2 \mathrm{MG} / \mathrm{c}^{2}$. It can be assumed that at the present time, $\mathrm{G}_{\mathrm{N}}(\mathrm{mm})=\mathrm{G}_{\mathrm{N}}$, and $\mathrm{G}_{\mathrm{N}}(\mathrm{dd}) \approx 10^{6} \mathrm{G}_{\mathrm{N}}$ because of the graviton evaporation in Fig. 1 . 
This condition indicates that the matters forms the black holes with the small Schwarzschild radius because of the small gravitation constant of $\mathrm{G}_{\mathrm{N}}(\mathrm{mm}) \approx \mathrm{G}_{\mathrm{N}}$. And, it can be assumed that always $\mathrm{k}(\mathrm{mm})=\mathrm{k} \approx 10^{48} \mathrm{k}(\mathrm{dd})$.

At the present time, $\mathrm{F}_{\mathrm{g}}(\mathrm{mm})=810^{-37} \mathrm{~F}_{\mathrm{c}}(\mathrm{mm}) \approx 10^{-36} \mathrm{~F}_{\mathrm{c}}(\mathrm{mm})$ for the proton. $\mathrm{F}_{\mathrm{c}}=\mathrm{F}_{\mathrm{c}}(\mathrm{EC})+\mathrm{F}_{\mathrm{c}}(\mathrm{LC})+\mathrm{F}_{\mathrm{c}}(\mathrm{CC}) \approx \mathrm{F}_{\mathrm{c}}(\mathrm{EC})$ $=\mathrm{k} \frac{E C 1 E C 2}{r^{2}}$ because $\mathrm{k}(\mathrm{EC})>\mathrm{k}(\mathrm{LC})>\mathrm{k}(\mathrm{CC})[3,4]$. The lepton charge force of $\mathrm{F}_{\mathrm{c}}(\mathrm{LC})$ plays an important role for the neutrinos with the zero EC charges and non-zero LC charges $[3,4]$. The missing neutrino fluxes can be studied again by using the lepton charge force of $\mathrm{F}_{\mathrm{c}}(\mathrm{LC})$ rather than the neutrino oscillation explanation. Here it is assumed that the $\mathrm{k}$ and $\mathrm{G}$ values are similar for the leptons and quarks. Then $\mathrm{F}_{\mathrm{c}}(\mathrm{mm}) \approx 10^{36} \mathrm{~F}_{\mathrm{g}}(\mathrm{mm}), \mathrm{F}_{\mathrm{g}}(\mathrm{dd})=10^{6} \mathrm{~F}_{\mathrm{g}}(\mathrm{mm})$ and $\mathrm{F}_{\mathrm{c}}(\mathrm{dd})=$ $10^{-12} \mathrm{~F}_{\mathrm{g}}(\mathrm{mm})$ for a proton-like particle in Fig. 1. This assumption can explain the relation of, at the present time, $\mathrm{F}_{\mathrm{c}}(\mathrm{mm})$ $>\mathrm{F}_{\mathrm{g}}(\mathrm{dd})>\mathrm{F}_{\mathrm{g}}(\mathrm{mm})>\mathrm{F}_{\mathrm{g}}(\mathrm{dm})>\mathrm{F}_{\mathrm{c}}(\mathrm{dd})>\mathrm{F}_{\mathrm{c}}(\mathrm{dm})=0$ for the proton-like particle in Fig. 1. For the B1 dark matter with the rest mass of $26.12 \mathrm{eV} / \mathrm{c}^{2}[1], \mathrm{F}_{\mathrm{g}}(\mathrm{dd}) \approx 10^{-10} \mathrm{~F}_{\mathrm{g}}(\mathrm{mm})$ and $\mathrm{F}_{\mathrm{c}}(\mathrm{dd})=\frac{4}{9} 10^{-12} \mathrm{~F}_{\mathrm{g}}(\mathrm{mm})$ where $\mathrm{F}_{\mathrm{g}}(\mathrm{mm})$ is for the proton. Therefore, $\mathrm{F}_{\mathrm{g}}(\mathrm{dd})>\mathrm{F}_{\mathrm{c}}(\mathrm{dd})$ for the B1, B2 and B3 dark matters as shown in Figs. 1 and 2. This assumption can explain the reason why the gravitational force strength $\left(\mathrm{F}_{\mathrm{g}}(\mathrm{mm})\right)$ between the matters is so weak compared with the electromagnetic force strength $\left(\mathrm{F}_{\mathrm{c}}(\mathrm{mm})\right)$ between the matters at the present time. Therefore, it is concluded that the Coulomb's constant is constant because of the photon confinement but the gravitation constant has been changed since the inflation because of the graviton evaporation along with the space evolution in Figs. 1 and 2 . It is expected that the changing process of the gravitation constant between the matters from $\mathrm{G}_{\mathrm{N}}(\mathrm{mm}) \approx 10^{36} \mathrm{G}_{\mathrm{N}}$ to $\mathrm{G}_{\mathrm{N}}(\mathrm{mm})=\mathrm{G}_{\mathrm{N}}$ happened mostly near the inflation period in Fig. 1. Therefore, during most of the universe evolution the gravitation constant could be taken as $G_{N}(m m)=G_{N}$. This explanation with the possible numerical values of $k$ and $G$ in Fig. 1 is only the example which needs to be further investigated in the future.

In Figs. 1 and 2, if the gravitons are confined within the corresponding space like the photons, the gravitation constant of $\mathrm{G}_{\mathrm{N}}(\mathrm{mm})$ could be larger than the gravitation constant of $\mathrm{G}_{\mathrm{N}}(\mathrm{dd})$ like $\mathrm{k}(\mathrm{mm})$ is larger than $\mathrm{k}(\mathrm{dd})$. In Figs. 1 and 2, it is proposed that $\mathrm{k}(\mathrm{dd})$ is much smaller than $\mathrm{k}(\mathrm{mm})$ in order to explain the charged dark matter distribution of the galaxy cluster. And if the gravitons are evaporated to other spaces, the gravitation constant of $\mathrm{G}_{\mathrm{N}}(\mathrm{mm})$ can be smaller than the gravitation constant of $\mathrm{G}_{\mathrm{N}}(\mathrm{dd})$. Experimentally, $\mathrm{F}_{\mathrm{g}}(\mathrm{mm})=8 * 10^{-37} \mathrm{~F}_{\mathrm{c}}(\mathrm{mm})$ for the proton. Therefore, the $\mathrm{G}_{\mathrm{N}}(\mathrm{mm})$ value is so small at the present time when compared with the $\mathrm{k}(\mathrm{mm})$ value. This indicates that the gravitons are evaporated as shown in Fig. 2. Because of the huge number $(\mathrm{N})$ of the evaporated gravitons into the $\mathrm{x} 1 \times 2 \times 3$ space in Figs. 1 and 2, the gravitational force between the dark matters on the $\mathrm{x} 1 \mathrm{x} 2 \mathrm{x} 3$ space should be stronger than the gravitational force between the matters of the baryons, leptons and mesons and the electromagnetic force between dark matters. Because of the strong gravitational force between the dark matters, the charged dark matters of the B1, B2 and B3 bastons are distributed following the gravitational forces rather than the electromagnetic force between the dark matters. The observed dark matter distributions around the galaxies and galaxy clusters support the strong gravitational force between the dark matters. As shown in Figs. 1 and 2, for the dark matters, $F_{g}(d d)>F_{c}(d d)$, for the matters $F_{g}(m m)$ $<<\mathrm{F}_{\mathrm{c}}(\mathrm{mm})$ and between the matter and dark matter $\mathrm{F}_{\mathrm{g}}(\mathrm{dm})>\mathrm{F}_{\mathrm{c}}(\mathrm{dm})=0$. Here $\mathrm{F}_{\mathrm{g}}$ and $\mathrm{F}_{\mathrm{c}}$ are the gravitational force strength and electromagnetic force strength, respectively. Also, it is assumed that $G_{N}(d d)>G_{N}(m m)>G_{N}(d m)$ for the gravitation constant and $\mathrm{k}(\mathrm{dm})=0, \mathrm{k}(\mathrm{mm})>>\mathrm{k}(\mathrm{dd})$ for the Coulomb's constant in Figs. 1 and 2. Here $\mathrm{d}$ and $\mathrm{m}$ mean the dark matter and (normal) matter, respectively. Then, $F_{c}(d m)=0, F_{c}(m m)>>F_{c}(d d)$ for the proton-like particle and $\mathrm{F}_{\mathrm{g}}(\mathrm{dm})<\mathrm{F}_{\mathrm{g}}(\mathrm{mm})<\mathrm{F}_{\mathrm{g}}(\mathrm{dd})$ for the proton-like particle. Also, $\mathrm{F}_{\mathrm{c}}(\mathrm{mm})>\mathrm{F}_{\mathrm{g}}(\mathrm{dd})$ in Figs. 1 and 2.

It has been observed from the gravitational lensing measurements for the bullet cluster [5], Abell 1689 cluster [6] and Abell 520 cluster [7] that the dark matters have been easily separated from the normal matters. The weak gravitational force with the small $\mathrm{G}_{\mathrm{N}}(\mathrm{dm})$ value between the dark matters and normal matters can explain why the dark matters are distributed as observed in the gravitational lensing measurements [5,6,7]. In other words, these gravitational lensing measurements $[5,6,7]$ are the direct evidence of the weak gravitational force with the small $\mathrm{G}_{\mathrm{N}}(\mathrm{dm})$ value between the dark matters and normal matters. Therefore, the dark matters and normal matters around the galaxies are connected by the weak gravitational force which can affect the rotational motions of the normal matters. For the bullet cluster [5], the dark matters and normal matters are taking the head and tail parts, respectively, when the corresponding galaxy cluster is moving. The strong gravitational force with the longer force range of the $g(0)$ graviton between the dark matters can make the location and shape of the dark matter distributions different from those of the normal matter distributions as observed in the Abell 1689 cluster [6] and Abell 520 cluster [7]. The weak gravitational force with the shorter force range of the $\mathrm{g}(0,0,0)$ graviton between the baryonic normal matters can make the location and the shape 
of the normal matter distributions as observed in the Abell 1689 cluster [6] and Abell 520 cluster [7], which have mostly the normal matters (galaxies) in the outside area and dark matters in the inside center area. Recently, the ultra-diffuse galaxy called as NGC1052-DF2 without the dark matters was found [8]. The formation of the galaxy without the dark matters could be explained with increasing of $F_{g}(\mathrm{dm})$ as a function of the time as shown in Figs. 1 and 3. The transition

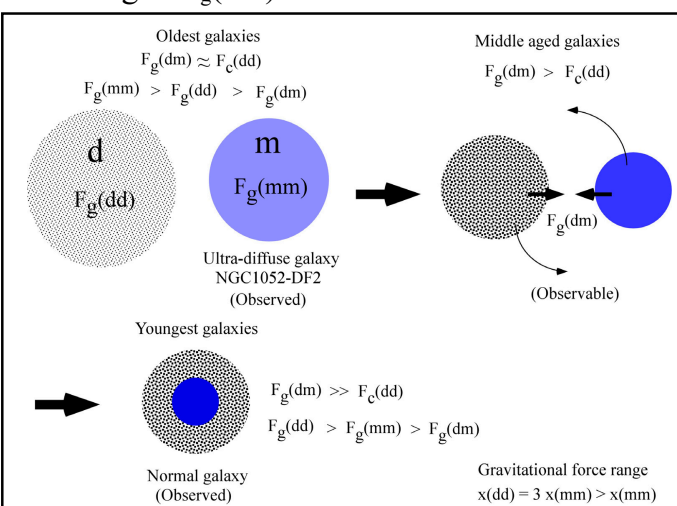

Fig. 3. The transition from the galaxy without the dark matters to the galaxy with the dark matters is shown (see Fig 1 and Table 1).

from the galaxy without the dark matters to the galaxy with the dark matters could be explained, too, as shown in Fig. 3. Therefore, the galaxies can be classified as the oldest galaxies, middle aged galaxies and the youngest galaxies as shown in Fig. 3. The ultra-diffuse galaxy called as NGC1052-DF2 without the dark matters was found [8] and is classified as the oldest galaxy. Then the dark matter galaxy and matter galaxy classified as the middle aged galaxies can rotate as the pair by the $\mathrm{F}_{\mathrm{g}}(\mathrm{dm})$ force. Therefore, looking for the rotating pair of the dark matter and matter galaxies will be interesting. See Ref. [9] for the gravitational force range for the dark matters in Fig. 3.

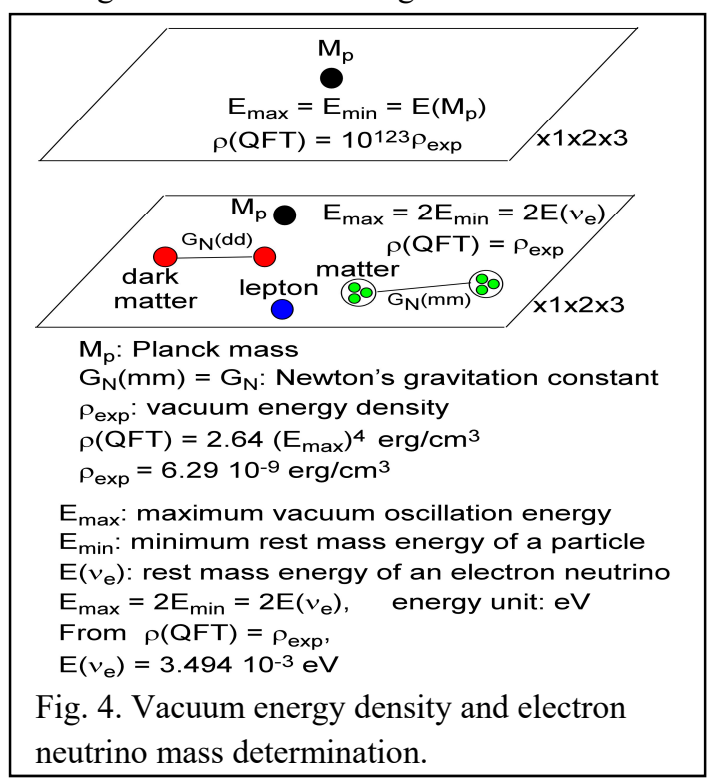

3. Dark energy, particle creation and gravitation constant

The dark energy can be described as the ground state oscillation of the vacuum energy. Then the vacuum energy density ( $\rho)$ was derived in terms of the QFT (quantum field theory) [10].

$$
\rho(Q F T)=\frac{\hbar}{2 \pi^{2} c^{3}} \int_{0}^{\omega_{\max }} \omega^{3} d \omega=\frac{\hbar}{8 \pi^{2} c^{3}} \omega_{\max }^{4}=\frac{1}{8 \pi^{2}(\hbar c)^{3}}\left(\hbar \omega_{\max }\right)^{4}
$$

The maximum vacuum oscillation energy is $\mathrm{E}_{\max }=\hbar \omega_{\max }$. When the Planck energy of $\mathrm{E}_{\mathrm{p}}=1.210^{19} \mathrm{GeV}$ is taken as the maximum vacuum oscillation energy, the obtained vacuum energy density is $\rho(Q F T)=5.8510^{114} \mathrm{erg} / \mathrm{cm}^{3} \mathrm{which}$ 
is too big to explain the observed vacuum energy density of $\rho_{\text {exp }}=6.2910^{-9} \mathrm{erg} / \mathrm{cm}^{3}$ [11]. The huge vacuum energy density of $\rho($ QFT $)=5.8510^{114} \mathrm{erg} / \mathrm{cm}^{3}$ is one of the unsolved questions in physics that is called as the cosmological constant puzzle.

This problem is solved in the present work as follows. In Fig. 4, the $\mathrm{x} 1 \mathrm{x} 2 \mathrm{x} 3$ space is the background space where the particles are moving. The vacuum energy is calculated based on the $\mathrm{x} 1 \mathrm{x} 2 \mathrm{x} 3$ space. The space oscillation energy can be converted to the rest masses of the elementary particles like the quarks, leptons and dark matters by the pair production of the particle and anti-particle in Fig. 4 when this space oscillation energy is greater than the rest mass energies of the created particles. Therefore, in the present work, the vacuum oscillation energy is defined as the space energy which cannot be larger than the rest mass energy of the created particle pair. From this new definition, the minimum rest mass energy of the particles can be taken as the half of the maximum vacuum oscillation energy of $\mathrm{E}_{\max }=\hbar \omega_{\max }$. In the $\mathrm{x} 1 \mathrm{x} 2 \mathrm{x} 3$ space without the elementary particles, the Planck energy of $\mathrm{E}_{\mathrm{p}}=1.210^{19} \mathrm{GeV}$ can be taken as the maximum vacuum oscillation energy in Fig. 4. The vacuum energy density of $\rho(Q F T)=5.8510^{114} \mathrm{erg} / \mathrm{cm}^{3}$ obtained on the $\mathrm{x} 1 \times 2 \times 3$ space without the elementary particles cannot explain the experimental vacuum energy density of $\rho_{\exp }=6.29$ $10^{-9} \mathrm{erg} / \mathrm{cm}^{3}$ observed on the $\mathrm{x} 1 \times 2 \times 3$ space with the elementary particles. And in the $\mathrm{x} 1 \times 2 \times 3$ space with the elementary particles of the dark matters (bastons), leptons and quarks, the rest mass energy of the electron neutrino ( $v_{\mathrm{e}}$ ) can be used as the half of the maximum vacuum oscillation energy of $E_{\max }=\hbar \omega_{\max }$ as shown in Fig. 4 . Therefore, from the relation of $\rho(Q F T)=\rho_{\exp }=6.2910^{-9} \mathrm{erg} / \mathrm{cm}^{3}$, the obtained rest mass energy of the electron neutrino $\left(v_{\mathrm{e}}\right)$ is $3.49410^{-3} \mathrm{eV}$. This one can be compared to the calculated one of $2.87610^{-7} \mathrm{eV}$ [1]. In other words, the rest mass of the electron neutrino $\left(v_{\mathrm{e}}\right)$ is determined to be $3.49410^{-3} \mathrm{eV} / \mathrm{c}^{2}$ experimentally from the observed vacuum energy density of $\rho_{\mathrm{exp}}=$ $6.2910^{-9} \mathrm{erg} / \mathrm{cm}^{3}[11]$ in terms of the quantum field theory (QFT).

In the present time, the Planck energy is $\mathrm{E}_{\mathrm{p}}(\mathrm{mm})=1.210^{19} \mathrm{GeV} \approx 10^{28} \mathrm{eV}$. All elementary particles can be created now because the Planck energy is bigger than the rest mass energies of the particles. For example, the proton was created from the vacuum fluctuations when the Planck energy is bigger than the rest mass energy of the proton. Then Planck energy is depending on the gravitation constant of $\mathrm{G}$. If $\mathrm{G}=10^{\mathrm{x}} \mathrm{G}_{\mathrm{N}}, \mathrm{E}_{\mathrm{p}}=(\hbar c / G)^{0.5}=10^{-\mathrm{x} / 2} 10^{28} \mathrm{eV}$. In Fig. 3 , when $E_{p} \geq E\left(v_{e}\right)$, the first electron neutrinos are created from the vacuum energy. From the relation of $E_{p}=10^{-2} \mathrm{eV}$, $\mathrm{G}_{\mathrm{N}}(\mathrm{mm})=10^{60} \mathrm{G}_{\mathrm{N}}$. Therefore, the electron neutrinos are the first particles created around $\mathrm{G}_{\mathrm{N}}(\mathrm{mm})=10^{60} \mathrm{G}_{\mathrm{N}}$ after big bang in Fig. 1 and Table 1. Here, from the relation of $E_{p} \geq E($ particle $)=10^{A} e V, A \leq 28-x / 2$. The first e, $u$ and $d$ quarks are created around $\mathrm{G}_{\mathrm{N}}(\mathrm{mm})=10^{44} \mathrm{G}_{\mathrm{N}}$ in Fig. 1 and Table 1 . And the first proton and neutron are created around $\mathrm{G}_{\mathrm{N}}(\mathrm{mm})=10^{36} \mathrm{G}_{\mathrm{N}}$ in Fig. 1 and Table 1 . And the $\mathrm{B} 1$ dark matter is created around $\mathrm{G}_{\mathrm{N}}(\mathrm{mm})=10^{52} \mathrm{G}_{\mathrm{N}}$ in Fig. 1 and Table 1. The B2 dark matter is created around $\mathrm{G}_{\mathrm{N}}(\mathrm{mm})=10^{34} \mathrm{G}_{\mathrm{N}}$ in Fig. 1 and Table 1. Therefore, the first Le, nuclei, gas and atoms could be formed roughly around $\mathrm{G}_{\mathrm{N}}(\mathrm{mm})=10^{30} \mathrm{G}_{\mathrm{N}}$. All elements with the rest masses less than $10^{22} \mathrm{eV}$ could be formed roughly around $\mathrm{G}_{\mathrm{N}}(\mathrm{mm})=10^{12} \mathrm{G}_{\mathrm{N}}$. The first galaxies and stars could be built around $\mathrm{G}_{\mathrm{N}}(\mathrm{mm})=10^{4} \mathrm{G}_{\mathrm{N}}$ in Fig. 1 and Table 1 at $\approx 10^{8}$ years after big bang according to the standard cosmological model. The rest mass of the Le particle is taken as $1.4 \mathrm{TeV}$ in Table 1 [9]. Dark matter galaxies are formed because the gravitational force between dark matters is greater than the electromagnetic force between dark matters in Fig. 1. The photon has the zero rest mass which means that the photon has been present since the big bang time when the gravitation constant is very large in Fig. 1 and Table 1. And the graviton could have the small rest mass of $3.1910^{-31} \mathrm{eV} / \mathrm{c}^{2}$ [9] which means that the graviton has been present since the big bang time when the gravitation constant is very large in Table 1 . The relations between particles, Planck energies and gravitation constants are listed in Table 1. It is expected that the changing process of the gravitation constant between the matters from $\mathrm{G}_{\mathrm{N}}(\mathrm{mm}) \approx 10^{36} \mathrm{G}_{\mathrm{N}}$ to $\mathrm{G}_{\mathrm{N}}(\mathrm{mm})=\mathrm{G}_{\mathrm{N}}$ happened mostly near the inflation period in Fig. 1 and Table 1 within $\approx 10^{9}$ years after big bang. And it is thought that the gravitation constant $\left(\mathrm{G}_{\mathrm{N}}(\mathrm{mm})\right)$ is nearly equal to the present gravitational constant $\left(\mathrm{G}_{\mathrm{N}}(\mathrm{mm})\right)$ of $\mathrm{G}_{\mathrm{N}}$ during most of the universe evolution.

Also, note that the vacuum energy can be transferred between dark matter space $(\mathrm{x} 1 \times 2 \times 3$ space $)$ and normal matter spaces $(x 4 \times 5 \times 6$ and $\times 7 \times 8 \times 9$ spaces $)$ in Fig. 2. The relations of $\mathrm{G}_{\mathrm{N}}(11)=\mathrm{G}_{\mathrm{N}}(\mathrm{qq})=\mathrm{G}_{\mathrm{N}}(\mathrm{mm})$ and $\mathrm{k}(11)=\mathrm{k}(\mathrm{qq})=\mathrm{k}(\mathrm{mm})=$ $\mathrm{k}$ are assumed in Fig. 1. Here, 1 and q represent the leptons and quarks. Then the normal matters consist of leptons, quarks and hadrons, and the dark matters are the B1, B2 and B3 bastons [2]. Also, near the inflation the relation of $E_{p}(m m) \geq E($ particle $)$ is used for the dark matter creation because $E_{p}(d d)>>E_{p}(m m)$ in Table 1. Therefore, all elementary particles including the B1, B2, B3 dark matters are created near the inflation as shown in Table. 1. The pair of the matter universe with the charge configuration of (EC, LC, CC) and anti-matter universe with the charge configuration of (-EC, -LC, -CC) could be created from the big bang because our universe is full of the matters. In this case, if the matter universe is defined to be negatively charged for the EC, LC and CC charges, the anti-matter universe should be defined to be positively charged for the EC, LC and CC charges. Then, the matters can be created from the 
decay of the matter universe with decreasing of the gravitation constant $(\mathrm{G}(\mathrm{mm}))$ in Fig. 1 and Table 1. Also, the pair of the matter and anti-matter can be created from the vacuum energy fluctuation with decreasing of the gravitation constant $(\mathrm{G}(\mathrm{mm}))$ in Fig. 1 and Table 1. The anti-particles created by the pair production of the particle and anti-particle are changed to the photons by the pair annihilation of the particle and anti-particle. And the particles created by the decay of the matter universe survive to form the galaxies and stars. This is why our matter universe is full of the particles. The decay of the matter universe to create the new particles takes place mostly near the inflation period through the formation of the universe particle and galaxy particles [9] in Table 1. But the pair production of the particle and antiparticle to be created from the vacuum energy fluctuation takes place always from the big bang time up to the present time. Also, the pair production of the matter universe and anti-matter universe can explain the CP symmetry problem of why the matters are dominating over the anti-matters on the present universe. And, the galaxy particles created through the decaying process of the matter universe should have the large black holes at the center and the particles at the outside like the Milky Way galaxy [9]. This is the reason why each galaxy has the large black hole at its center.

\section{Effective mass and effective charge}

In Figs. 1 and 2, the Coulomb's constant $(\mathrm{k})$ and gravitation constant $(\mathrm{G})$ have been changed in terms of the conserved charges and conserved rest masses of the particles. Then the effective charges and effective rest masses of the particles can be defined in terms of the fixed Coulomb's constant $(\mathrm{k})$ and fixed gravitation constant $\left(\mathrm{G}_{\mathrm{N}}\right)$. For example, $\mathrm{F}_{\mathrm{c}}(\mathrm{EC})$ $=\mathrm{k} \frac{E C 1 E C 2}{r^{2}}$ for the matters and and $\mathrm{F}_{\mathrm{c}}(\mathrm{EC})=10^{-48} \mathrm{k} \frac{E C 1 E C 2}{r^{2}}=\mathrm{k} \frac{\left(10^{-24} E C 1\right)\left(10^{-24} E C 2\right)}{r^{2}}=\mathrm{k} \frac{(E C 1)_{e f f}(E C 2)_{e f f}}{r^{2}}$ for the dark matters. So the effective charge of the dark matter is $(E C)_{\text {eff }}=10^{-24} E C$. For example, the effective charge of the B1 dark matter with the $\mathrm{EC}=-\frac{2}{3} \mathrm{e}$ is $(\mathrm{EC})_{\mathrm{eff}}=-\frac{2}{3} 10^{-24} \mathrm{e}$. Also, at the present time $\mathrm{F}_{\mathrm{g}}(\mathrm{mm})=\mathrm{G}_{\mathrm{N}} \frac{m 1 m 2}{r^{2}}$ for the matters and and $\mathrm{F}_{\mathrm{g}}(\mathrm{dd})=10^{6} \mathrm{G}_{\mathrm{N}} \frac{m 1 m 2}{r^{2}}=\mathrm{G}_{\mathrm{N}} \frac{\left(10^{3} m 1\right)\left(10^{3} m 2\right)}{r^{2}}=G_{N} \frac{(m 1)_{e f f}(m 2)_{e f f}}{r^{2}}$ for the dark matters. So the effective rest mass of the dark matter is $m_{\text {eff }}=10^{3} \mathrm{~m}$. For example, the effective rest mass of the $\mathrm{B} 1$ dark matter with the rest mass of $26.12 \mathrm{eV} / \mathrm{c}^{2}[1]$ is $\mathrm{m}_{\mathrm{eff}}=26.1210^{3} \mathrm{eV} / \mathrm{c}^{2}$. The first gas and stars existed at $1.810^{8}$ years after big bang [12]. It was reported $[12,13]$ that the baryon gas temperature at that time was lower than expected by the standard cosmological model. And the first gas and stars could be created when the gravitation constant of the matters is $10^{4} \mathrm{G}_{\mathrm{N}}$ in Table 1 and Fig. 2. Therefore, the colder gas temperature could be related with the strong gravitation constant of $10^{4} \mathrm{G}_{\mathrm{N}}$ which increases the potential energy and decreases the kinetic energy compared with the gas temperature with the current gravitation constant of $\mathrm{G}_{\mathrm{N}}$. In other words, the effective rest masses of the baryons should be increased by the factor of $10^{2}$ at $310^{8}$ years after the big bang compared with the rest masses of the baryons with the current gravitation constant of $\mathrm{G}_{\mathrm{N}}$. The large effective rest masses of the baryons and the large gravitation constant between the baryons could explain the cooler gas temperature measured by the EDGE experiments at 2-3 10 $0^{8}$ years after big bang [12].

\section{Summary}

In the present work, the dark matter properties of the bastons are shown for the gravitational force and electromagnetic force. When the proper values of the gravitation constants and Coulomb's constants are given for the normal matter and dark matters, the charged dark matters like the B1, B2 and B3 dark matters can be the good candidates of the dark matters. The B1, B2 and B3 bastons are originated from the extended standard model which is called as the threedimensional quantized space model. Here it is assumed that the $\mathrm{k}$ and $\mathrm{G}$ values are similar for the leptons and quarks. Then $\mathrm{F}_{\mathrm{c}}(\mathrm{mm}) \approx 10^{36} \mathrm{~F}_{\mathrm{g}}(\mathrm{mm}), \mathrm{F}_{\mathrm{g}}(\mathrm{dd})=10^{6} \mathrm{~F}_{\mathrm{g}}(\mathrm{mm})$ and $\mathrm{F}_{\mathrm{c}}(\mathrm{dd})=10^{-12} \mathrm{~F}_{\mathrm{g}}(\mathrm{mm})$ for a proton-like particle in Fig. 1. This assumption can explain the relation of, at present time, $F_{c}(\mathrm{~mm})>F_{g}(d d)>F_{g}(m m)>F_{g}(d m)>F_{c}(d d)>F_{c}(d m)=0$ for the proton-like particle in Fig. 1 . For the $\mathrm{B} 1$ dark matter with the rest mass of $26.12 \mathrm{eV} / \mathrm{c}^{2}[1], \mathrm{F}_{\mathrm{g}}(\mathrm{dd}) \approx 10^{-10} \mathrm{~F}_{\mathrm{g}}(\mathrm{mm})$ and $F_{c}(d d)=\frac{4}{9} 10^{-12} F_{g}(\mathrm{~mm})$ where $F_{g}(\mathrm{~mm})$ is for the proton. Therefore, $F_{g}(d d)>F_{c}(d d)$ for the B1, B2 and B3 dark matters as shown in Figs. 1 and 2. In order to make dark matters to be controlled by the attractive gravitational force, the attractive gravitational force $\left(\mathrm{F}_{\mathrm{g}}(\mathrm{dd})\right)$ between the dark matters should be greater than the repulsive Coulomb's force $\left(F_{c}(d d)\right)$ between the dark matters. Therefore, $F_{g}(d d)>F_{c}(d d)$. Therefore, the relation of $F_{c}(m m)>F_{g}(d d)>$ $F_{g}(m m)>F_{g}(d m)>F_{c}(d d)>F_{c}(d m)=0$ for the proton-like particle is obtained as shown in Fig. 1. Therefore, it is concluded that the Coulomb's constant is constant because of the photon confinement but the gravitation constant has been changing since the inflation because of the graviton evaporation along with the space evolution in Fig. 1. It is expected that the changing process of the gravitation constant between the matters from $\mathrm{G}_{\mathrm{N}}(\mathrm{mm}) \approx 10^{36} \mathrm{G}_{\mathrm{N}}$ to $\mathrm{G}_{\mathrm{N}}(\mathrm{mm})$ 
$=\mathrm{G}_{\mathrm{N}}$ happened mostly near the inflation period in Fig. 1 . Therefore, during the most of the universe evolution the gravitation constant could be taken as $\mathrm{G}_{\mathrm{N}}(\mathrm{mm})=\mathrm{G}_{\mathrm{N}}$. This explanation with the possible numerical values of $\mathrm{k}$ and $\mathrm{G}$ in Fig. 1 is only the example which needs to be further investigated in the future. This assumption can explain the reason why the gravitational force strength $\left(\mathrm{F}_{\mathrm{g}}(\mathrm{mm})\right)$ between the matters is so weak compared with the electromagnetic force strength $\left(\mathrm{F}_{\mathrm{c}}(\mathrm{mm})\right)$ between the matters.

The relations between particles, Planck energies and gravitation constants are listed in Table 1. It is expected that the changing process of the gravitation constant between the matters from $\mathrm{G}_{\mathrm{N}}(\mathrm{mm}) \approx 10^{36} \mathrm{G}_{\mathrm{N}}$ to $\mathrm{G}_{\mathrm{N}}(\mathrm{mm})=\mathrm{G}_{\mathrm{N}}$ happened mostly near the inflation period in Fig. 1 and Table 1 . And it is thought that the gravitational constant $\left(\mathrm{G}_{\mathrm{N}}(\mathrm{mm})\right)$ is nearly equal to the present gravitational constant $\left(\mathrm{G}_{\mathrm{N}}(\mathrm{mm})\right)$ of $\mathrm{G}_{\mathrm{N}}$ during most of the universe evolution. The particles created by the decay of the matter universe survive to form the galaxies. This is why our matter universe is full of the particles. The pair production of the matter universe and anti-matter universe can explain the CP symmetry problem of why the matters are dominating over the anti-matters on the present universe [4,9]. And, the galaxy particles created through the decaying process of the matter universe should have the large black holes at the center and the particles at the outside like the Milky Way galaxy [9]. This is the reason why each galaxy has the large black hole at its center. In Figs. 1 and 2, the Coulomb's constant (k) and gravitation constant $(\mathrm{G})$ have been changed in terms of the conserved charges and conserved rest masses of the particles. And the effective charges and effective rest masses of the particles are defined in terms of the fixed Coulomb's constant $(\mathrm{k})$ and fixed gravitation constant $\left(\mathrm{G}_{\mathrm{N}}\right)$. The effective charge of the $\mathrm{B} 1$ dark matter with the $\mathrm{EC}=-\frac{2}{3} \mathrm{e}$ is $(\mathrm{EC})_{\mathrm{eff}}=-\frac{2}{3} 10^{-24} \mathrm{e}$. And the large effective rest masses of the baryons and the large gravitation constant between the baryons could explain the cooler gas temperature measured by the EDGE experiments at 2-3 $10^{8}$ years after big bang [12].

\section{References}

[1] R. Barkana et al., arXiv:1803.03091v2 (2018).

[2] Jae-Kwang Hwang, Mod. Phys. Lett. A32, 1730023 (2017)

[3] Jae-Kwang Hwang, https://www.researchgate.net/publication/313247136

[4] Jae-Kwang Hwang, https://www.researchgate.net/publication/297270485

[5] D. Clower et al., Astrophys. J. 604, 596 (2004).

[6] A.N. Taylor et al., Astrophys. J. 501, 539 (1998).

[7] M. Jee et al., Astrophys. J. 783, 1 (2014).

[8] P. van Dokkum et al., Nature 555, 629 (2018).

[9] Jae-Kwang Hwang, https://www.researchgate.net/publication/325553139

[10] S.E. Rugh and H. Zinkernagel, arXiv:hep-th/0012253 (2000).

[11] https://map.gsfc.nasa.gov/

[12] J.D. Bowman et al., Nature 555, 67 (2018).

[13] J.B. Munoz and A. Loeb, Nature 557, 684 (2018). 